\title{
Effect of Surrounding Gas on Cavitation in EHL
}

\author{
Takefumi Otsu ${ }^{1)^{*}}$, Hiroyoshi Tanaka ${ }^{2)}$, Naoshi Izumi ${ }^{2)}$ and Joichi Sugimura ${ }^{2)}$ \\ ${ }^{1)}$ Department of Mechanical Engineering Science, Graduate School of Engineering, Kyushu University \\ 744 Motooka, Nishi-ku, Fukuoka 819-0395, Japan \\ ${ }^{2)}$ Department of Mechanical Engineering, Kyushu University \\ 744 Motooka, Nishi-ku, Fukuoka 819-0395, Japan \\ *Corresponding author: te207299@s.kyushu-u.ac.jp
}

( Manuscript received 2 February 2009; accepted 9 March 2009; published 16 April 2009 )

(Presented at JAST Tribology Conference Nagoya, September 2008)

\begin{abstract}
Relationship between cavitation in elastohydrodynamic lubrication and surrounding gas was investigated in order to understand lubrication in various gas environments. Lubricated point contact sliding test was conducted in a sealed chamber. Gases used were air, carbon dioxide, argon and helium. It was found the cavity length increased with time. It was also found that the cavity length in gas with higher gas solubility, such as carbon dioxide, was larger than that in gas with lower gas solubility. These results suggested that dissolved gas in lubricant was gradually released into the cavity with time, and the cavity length depended on the amount of dissolved gas in the lubricant.
\end{abstract}

Keywords: elastohydrodynamic lubrication, cavitation, gas, dissolution, carbon dioxide

\section{Introduction}

Machine elements often have to work in environments other than ambient air. It is well recognized that surrounding gas affects lubrication performance in any lubrication regimes. In hydrodynamic lubrication, surrounding gas and its pressure affect dissolution of the gas itself into lubricant, which may cause changes in viscosity of the lubricant, and may further cause degradation of the lubricant. Therefore, investigating effects of surrounding gas on lubrication is important for establishing design for reliability and safety.

Cavitation in hydrodynamic lubrication is vaporization of lubricant or release of dissolved gas in lubricant ${ }^{1)}$.Outlet cavity in lubricant film may cause starvation of lubricant and even breakdown of lubricating film in reciprocating contact. Nishikawa et al. reported oil starvation in reciprocating sliding tests, and suggested that cavity formed at the exit of conjunction affected formation of lubricant film in the next stroke ${ }^{2)}$. More recently, Leonard et al. reported that gaseous cavities affected fretting wear under grease lubricated conditions $^{3)}$. On the other hand, cavitation has a positive effect of reducing friction ${ }^{4)}$.

Considering the gaseous cavitation, the kind of surrounding gas must be related with cavitation, because the amount of dissolved gas in lubricant is different for different gases. For example, time for lubricant film to break down in HFC134a, a refrigerant gas, was shorter than that in nitrogen gas ${ }^{5}$. This suggested that starvation in HFC134a was larger than that in nitrogen, implying that cavity in HFC134a was greater than that in nitrogen, and this was caused by difference in the gas solubility of HFC134a and that of nitrogen ${ }^{6}$.

In this study, point contact EHL experiments are conducted in some gases as well as in vacuum in order to find the relationship between surrounding gas and behavior of the outlet cavitation.

\section{Experimental}

2.1. Test apparatus and conditions

Figure 1 schematically shows test apparatus used in this study. Lubricated point contact sliding tests were conducted in a sealed chamber, which was filled with arbitrary test gas.

A plano convex glass lens was pressed against a glass disc by a lever while the disc was rotated by a motor. The glass disc and the glass lens were made of BK7, optical glass. The diameter and the thickness of the disc were $80 \mathrm{~mm}$ and $8 \mathrm{~mm}$, respectively. Radius of curvature of the lens was $10.38 \mathrm{~mm}$. The disc was coated with semi-reflective chromium layer and silica overlay for measuring lubricant film thickness with ultra-thin film interferometry ${ }^{7}$. The lens was coated with chromium for full reflection. 


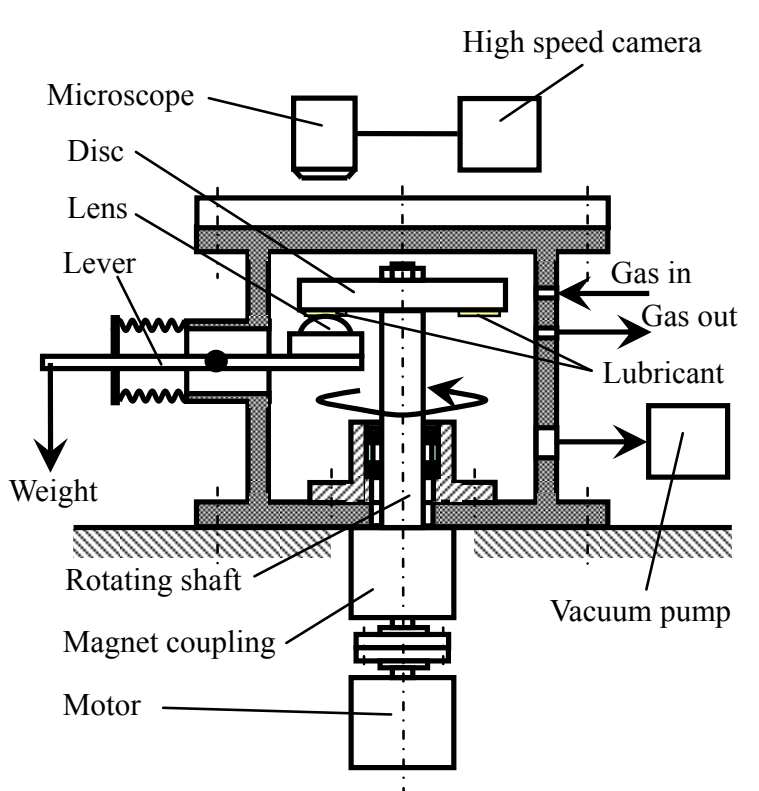

Fig.1 Test apparatus

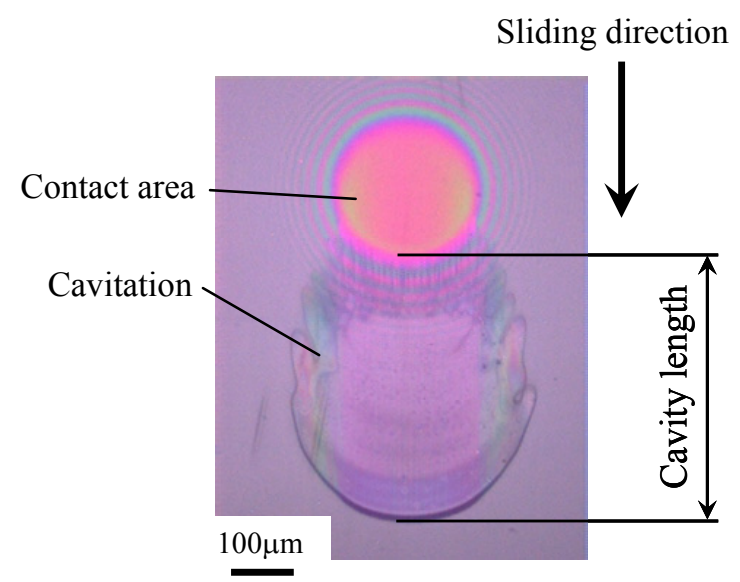

Fig.2 Definition of cavity length

A vacuum pump was connected to the chamber. The chamber had a gas port in order to introduce gas after vacuuming. Contact area between the disc and the lens was observed by a microscope from a view port located on a chamber lid. A high speed video camera was connected to the microscope in order to record the conjunction, for determining EHL film thickness and the cavity length.

Sliding tests were conducted at a normal load of $7 \mathrm{~N}$, the corresponding maximum Hertzian pressure being $0.27 \mathrm{GPa}$, and at a sliding speed of $20 \mathrm{~mm} / \mathrm{s}$. Gas used was air, carbon dioxide, argon and helium. Polyalphaolefin, PAO, with a viscosity of $63 \mathrm{~mm}^{2} / \mathrm{s}$ at $313 \mathrm{~K}$, was used as a lubricant in this study. An amount of $0.2 \mathrm{ml}$ of the lubricant was supplied on the disc surface before setting the disc. The tests were conducted under ambient temperature of $295 \mathrm{~K}$.

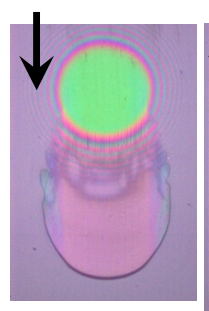

(a) $10 \mathrm{sec}$

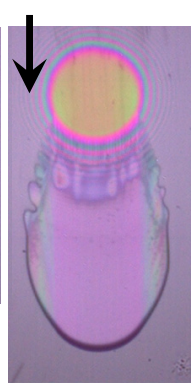

(b) $1 \mathrm{~min}$

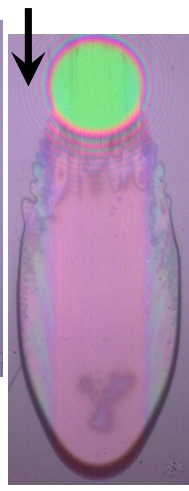

(c) $10 \mathrm{~min}$

Fig.3 Growth of cavity

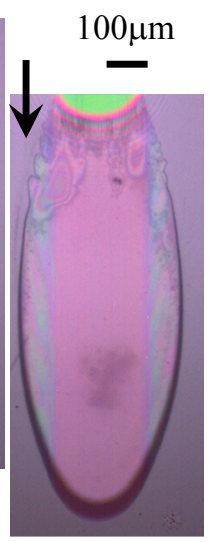

(d) $30 \mathrm{~min}$

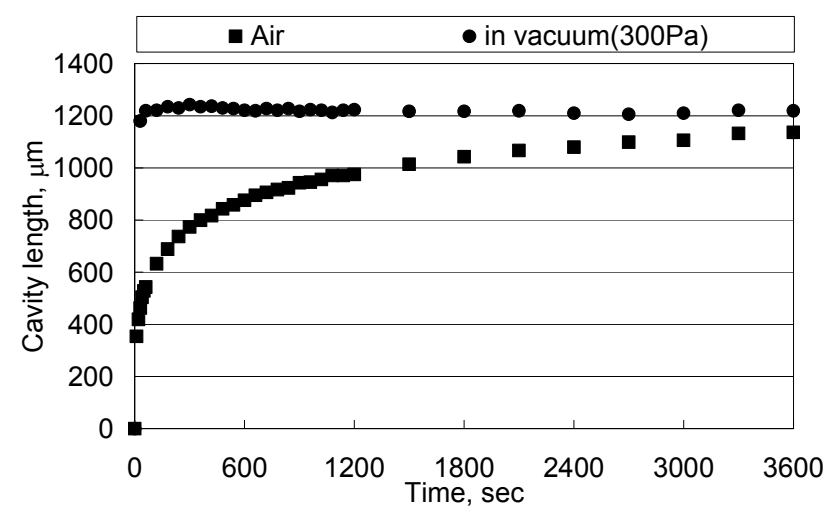

Fig.4 Changes in cavity length with time

\subsection{Definition of cavity length}

Figure 2 shows a photograph of an EHL conjunction. Hertzian contact area is clear by the interference pattern, and cavity at the exit of conjunction is also visible. In order to study size of the cavities, cavity length is defined as a distance from the edge of Hertzian contact area to the trailing edge of the cavity.

\section{Results and discussion}

\subsection{Time dependence of cavity length}

Figure 3 shows photographs of cavity at 10 seconds, 1 minute, 10 minutes and 30 minutes from the start of the test in air. The arrow in the figure shows direction of flow. Figure 4 shows changes in the cavity length with time in air and in vacuum conditions.

These results clearly demonstrate that cavity gradually grows with time. Figure 3 shows that cavity grows to the direction of flow. Figure 4 shows that the cavity length rapidly increases from the start of the tests to about 600 seconds. After about 600 seconds, the cavity gradually grows with time.

Temperature of the lubricant at the conjunction exit was measured with thermocouple. It was found that, under the present test conditions, no appreciable temperature rise occurred. 


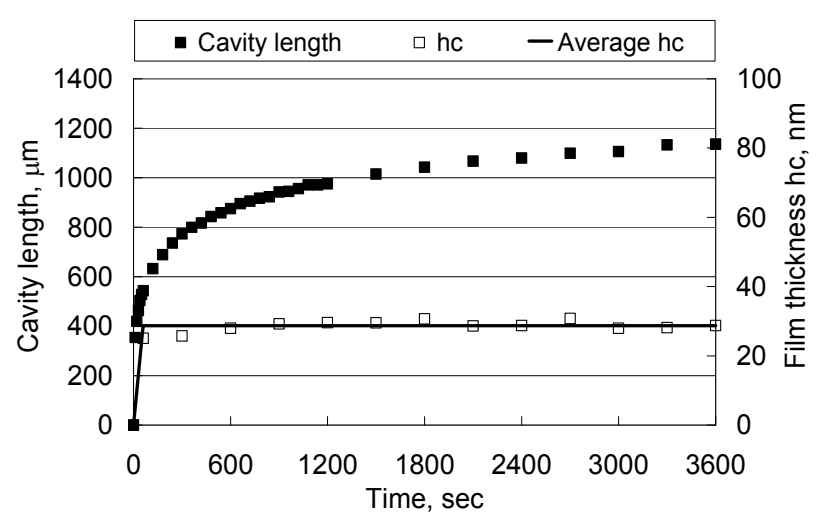

Fig.5 Relation between cavity length and film thickness in air
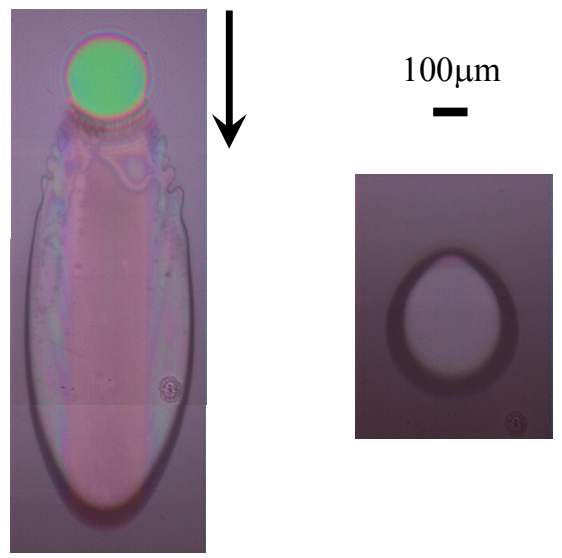

Fig.6 Cavity at 30 minutes (left) and at 0.3 seconds after motion is stopped (right)

Figure 5 shows the cavity length and the central film thickness determined by the ultra-thin film interferometry. The average central film thickness was $28.7 \mathrm{~nm}$. The central film thickness almost did not change during the test. Small fluctuation in the film thickness may be due to uneven coating film thickness.

Figure 6 shows photographs of cavity at 30 minutes and bubble remained after sliding motion is stopped. The average diameters of the bubble at 0.3 seconds and that at 60 minutes after the stop were $406 \mu \mathrm{m}$ and 276 $\mu \mathrm{m}$, respectively. For the cavity to last long after the motion is stopped, the well grown cavity must contain a larger amount of gas with higher pressure than the pressure it had when the cavity was initially formed. Therefore, it is likely that the growth of the cavity is caused by gradual release of dissolved gas in the lubricant into the cavity.

In Fig. 4, the circle symbol shows the cavity length in vacuum. Pressure in the chamber during vacuuming was about $300 \mathrm{~Pa}$. The test in the vacuum condition was conducted after vacuuming for three hours. The result indicates that the cavity length in vacuum is larger than

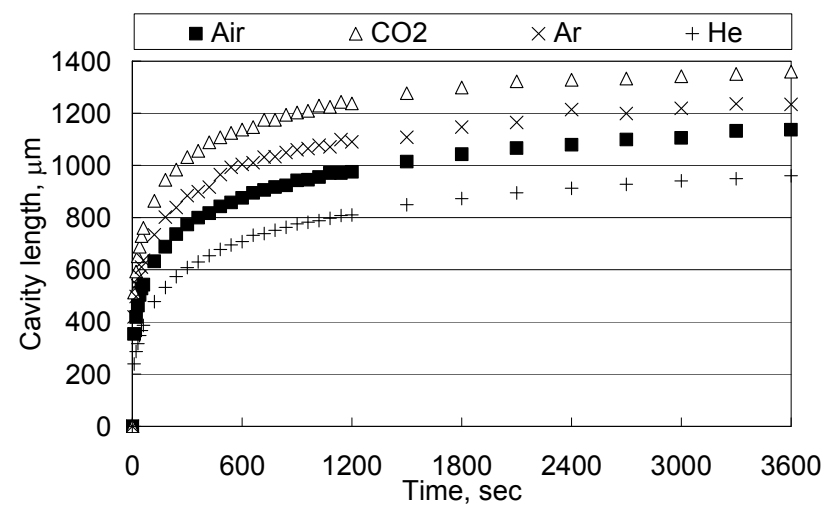

Fig.7 Changes in cavity length

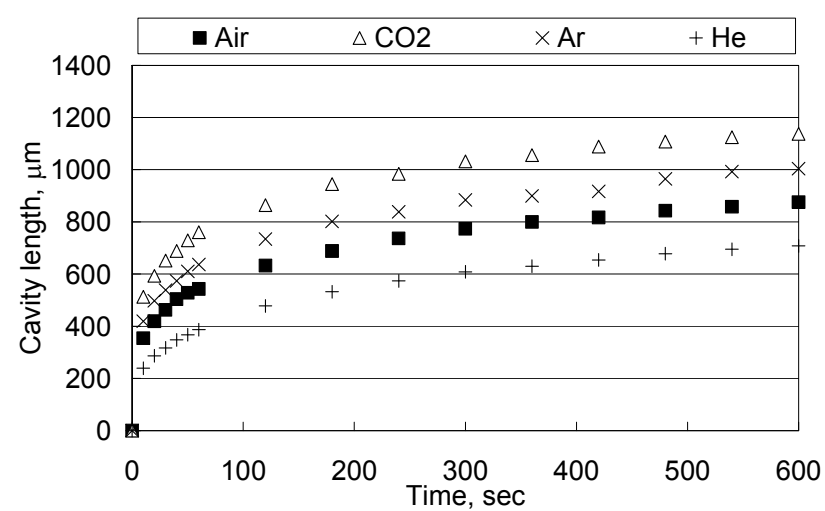

Fig. 8 Changes in cavity length to 600 seconds

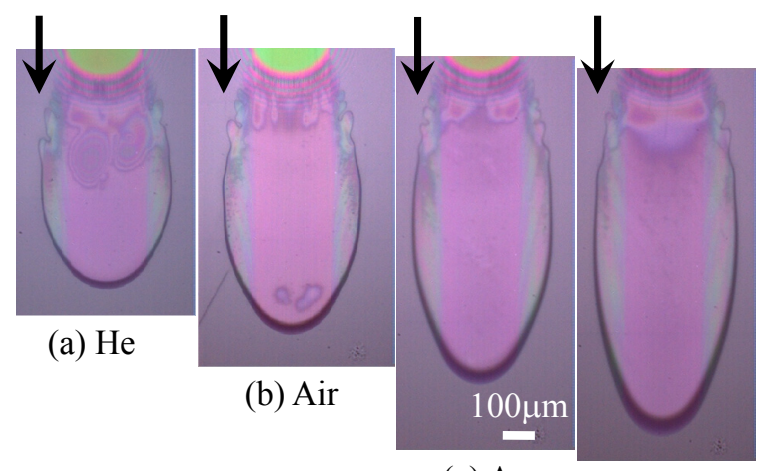

(c) $\mathrm{Ar}$

(d) $\mathrm{CO}_{2}$

Fig.9 Cavity at 300 seconds

that in air. This is because cavitation occurs more easily at lower background pressure.

The figure also clearly shows that no change in cavity length occur in vacuum. This is due to little dissolved gas in the lubricant and no dissolution of surrounding gas into the lubricant by vacuuming. From the results in air and in vacuum, it is concluded that the cavity growth with time relates to the release of dissolved gas. 


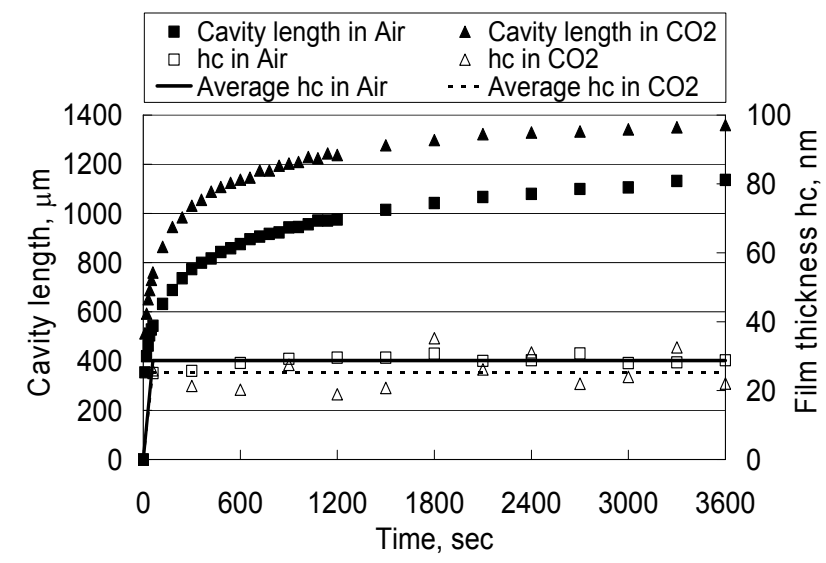

Fig.10 Cavity length and film thickness in air and carbon dioxide

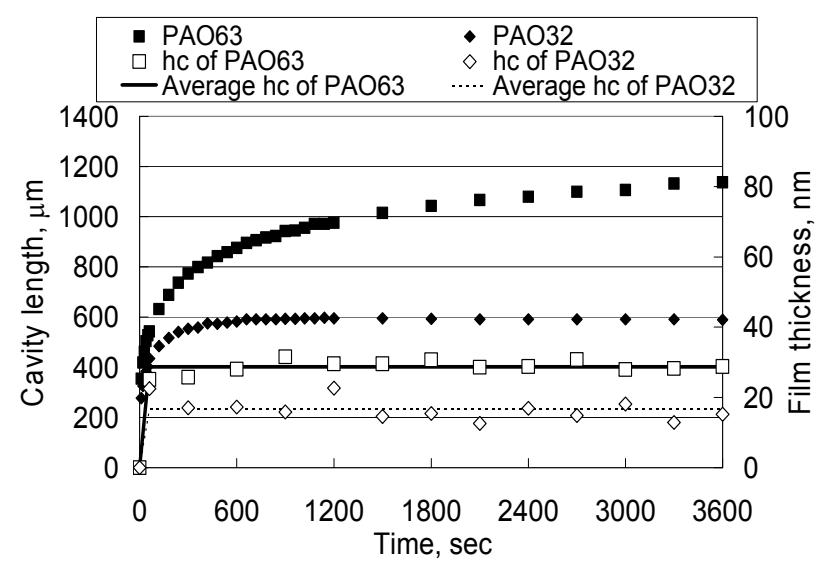

Fig.11 Effect of lubricant viscosity on cavity length

\subsection{Effect of surrounding gas on cavity length}

Gas in the chamber was controlled by the following method. The test gas was introduced to atmospheric pressure after vacuuming for three hours. Pressure in the chamber during vacuuming was about $300 \mathrm{~Pa}$.

Figure 7 shows changes in cavity length from the start of the test to one hour. Figure 8 shows changes in the cavity length for 600 seconds. Figure 9 compares the cavities at 300 seconds.

These figures clearly demonstrate that the cavity length is different for different kind of the gas. The cavity length in carbon dioxide is the largest among the tested gases, and that in helium is the smallest. Figure 8 shows that difference in the cavity length for different kinds of gases is observed from the onset of the tests. Figure 9 shows that the cavity grew in the direction of flow in all the cases.

Figure 10 shows the cavity length and the central film thickness in air and carbon dioxide. The central film thickness in carbon dioxide is slightly smaller than that in air. The reason for this may be decrease in viscosity of the lubricant due to dissolution of carbon dioxide during the test.

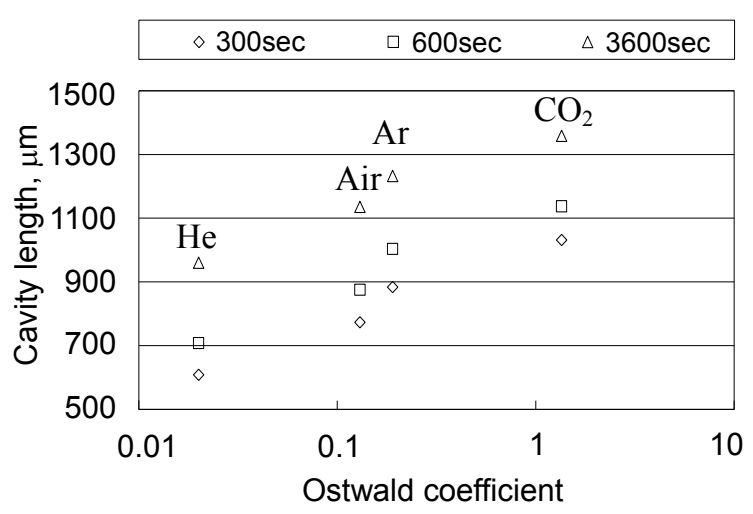

Fig.12 Relation between cavity length and gas solubility

Thicker oil generally gives rise to longer cavity under the same speed and load due to broader hydrodynamic pressure generation, as shown in Fig. 11 in which PAO63 gives longer cavity than PAO32, with lower viscosity of $33 \mathrm{~mm}^{2} / \mathrm{s}$ at $313 \mathrm{~K}$. However the cavity length in carbon dioxide is larger than that in air in spite of the reduced film thickness in carbon dioxide. Therefore, the longer cavity in carbon dioxide should be ascribed to release of dissolved gas into the cavity.

Figure 12 shows relationship between the cavity length and gas solubility. The cavity lengths at 300 seconds, 600 seconds and 3600 seconds are shown in the figure. Ostwald coefficient for gas solubility is calculated by ASTM D $2779^{8)}$. The cavity length increases with gas solubility, and this strongly suggests that the cavity length depends on the amount of dissolved gas. The amount of gas released in cavity should increase with the amount of dissolved gas. Therefore, the cavity length in the gas with higher gas solubility is larger.

\section{Conclusions}

The following conclusions are drawn from this study.

(1) The cavity length increases with time.

(2) The cavity length is affected by surrounding gas. The cavity length in gas with higher gas solubility is larger.

\section{Acknowledgement}

The authors are grateful to Idemitsu Kosan Co., Ltd. for supplying the lubricants.

\section{References}

[1] Dowson, D. and Taylor, C. M., "Fundamental Aspects of Cavitation in Bearings," In "Cavitation and Related Phenomena in Lubrication," Ed. Dowson et al., Mechanical Engineering Publications Ltd., Bury St Edmunds, 1975, 15-26. 
[2] Nishikawa, H., Handa, K. and Kaneta, M., "Behavior of EHL Films in Reciprocating Motion," JSME International Journal, Series C, 38 , 3, 1995, 558-567.

[3] Leonard, B., Sadeghi, F. and Cipra, R., "Gaseous Cavitation and Wear in Lubricated Fretting Contacts," Tribology Transactions, 51, 2008, 351-360.

[4] Yagi, K., Takedomi, W., Tanaka, H. and Sugimura, J., "Improvement of Lubrication Performance by Micro Pit Surfaces," Tribology Online, 3, 5, 2008, 285-288.

[5] Yamamoto, Y., Kim, J., Gondo, S. and Sugimura, J., "The Effect of HFC Refrigerant Dissolution on Oil Film Thickness and Wear Characteristics of
Oils," In "Thinning Films and Tribological Interfaces," Ed. Dowson et al., Elsevier B. V., Amsterdam, 2000, 771-776.

[6] Yamamoto, Y., Gondo, S., and Kim, J., "Solubility of HFC134a in Lubricants and Its Influence on Tribological Performance," Tribology Transactions, 44, 2, 2001, 209-214.

[7] Johnston, G. J., Wayte, R. and Spikes, H. A., "The Measurement and Study of Very Thin Lubricant Films in Concentrated Contacts," Tribology Transactions, 34, 1997, 187-194.

[8] Hata, H., "Solubility of Gases in Lubricants and Effect of Dissolved Gases on Physical Properties of Lubricants (1)," Journal of Japanese Society of Tribologists, 35, 7, 1990, 471-474 (in Japanese). 